\title{
ACQUIRE HIGH QUALITY MESHES OF SCALE MODELS FOR AN AUTOMATIC MODELLING PROCESS
}

\author{
F. Giraud, K. Jacquot, C. Chevrier, G. Halin
}

\author{
MAP-CRAI, National School of Architecture of Nancy, 2 rue Bastien Lepage, 54000 Nancy, France \\ (giraud, jacquot, chevrier, halin)@crai.archi.fr
}

KEY WORDS: 3D Modelling, Scale Models, Photogrammetry, Laser Scan, Segmentation

\begin{abstract}
:
Urban scale models depicting whole towns such as the hundred-scale model collection known as plans-reliefs are a valuable source of information of cities and their surroundings. These physical representations of French strongholds from the $17^{\text {th }}$ through the $19^{\text {th }}$ century suffer from many problems that are, among other things, wear and tear or the lack of visibility and accessibility. A virtual collection would allow remote accessibility for visitors as well as history researchers. Moreover, it may also be linked to other digital collections and therefore, promote the collection to make people come to the museums to see the physical scale models. We also work on other physical town scale models like Epinal for which the scale is a bit higher.
\end{abstract}

In a first part, we define a protocol for acquiring 3D meshes of town scale models from both photogrammetric and scanning methods. Then we compare the results of both methods The photogrammetric protocol has been elaborated by choosing the most accurate software, 123DCatch, which asks for about 60 pictures, and defining the settings needed to obtain exploitable photographs. In the same way, we defined the devices and settings needed for the laser scan acquisition method. In a second part, we segment the 3D meshes in planes by using Geomagic, which has been chosen between several programs, for its accurate resulting geometry.

\section{INTRODUCTION}

Urban scale models depicting whole towns such as the hundredscale models collection known as plans-reliefs are a valuable source of information of cities and their surroundings. These physical representations of French strongholds from the $17^{\text {th }}$ through the $19^{\text {th }}$ century suffer from many problems that are, among other things, wear and tear or the lack of visibility and accessibility. Nevertheless, they are objects that come back with a new visibility to the public, with the apparition of the computer tools, and the new interest for city history. This can be seen by the new wave of copies of scale models, and the new exhibitions in which they are highlighted. To grant an access to a larger public, some researchers work on the possibilities to recreate them faithfully. A virtual collection would allow remote accessibility for visitors as well as history researchers. Moreover, it may also be linked to other digital collections and therefore, promote the collection to make people come to the museums to see the physical scale models.

In this paper, we focus on the very first steps in the $3 \mathrm{D}$ digitising/modelling pipeline that are the raw data acquisition and processing. 3D data acquisition from scale models is a challenging task for many reasons: the level of detail of the scale models, the high accuracy needed for modelling, the limited access to scale models, and the low luminous conditions in which they are exposed. We have been experimenting different acquisition methods, and we defined a full operation protocol, in order to automate the process. We will first introduce the town scale models, their specificities, and their complexities in comparison to the full-scale models (section 2). Then related works are presented (section 3), in terms of town scale model modelling, and comparisons between photogrammetrical and 3D laser scanning methods. Later, we will determinate the most appropriate software and method for high quality meshes acquisition, and define a protocol for each of these methods, by explaining our experiments (sections 4 and 5). One of these methods will be chosen (section 6). It will be followed by our experiments on segmentation of these meshes (section 7). And finally we will conclude with a presentation of our results (section 8 ).

\section{SCALE MODELS}

The town scale model tradition began in the $17^{\text {th }}$ century. In France, a collection of 144 scale models has been initiated by Vauban, named "Plans-Reliefs". They were made at a scale of $1 / 600$, and their average size often approaches 50 square meters. Most of the old scale models are damaged by the time, and are difficult to move. In order to protect them and to grant a larger access to these pieces of history, there is a real interest to create 3D semantic models of the scale models that would be available for viewing by the public on the Internet. In parallel to these olds scale models and collections, a new wave of freshly realised scale models is actually coming. They sometimes have different scales, as Epinal's one (1/300 ${ }^{\circ}$ scale).

In order to acquire their 3D meshes, there are many differences between a scale model and a full-scale object. The acquisition of a scale model needs much more precision than the full-scale object. Indeed, they have the same details of architectural complexity, only represented much smaller. 10 centimetres long in real life are represented by only a half-millimetre on the scale model. When the streets are 5 to 10 metres wide, they only measure 1 to 2 centimetres. The 3D acquisition of town scale models is much more difficult, and needs to be precise. The scale model reconstruction is a real difficult work. From this point of view, this operation needs some preparation, and a protocol must be defined in order to realise the acquisition in optimal conditions.

\section{RELATED WORKS}

\subsection{Town scale model acquisition and modelling}

Town scale model acquisition and remodelling has already been the subject of many studies. Most of these experiments use the scan data only as a support, to manually model the object. The 
Project Relief Auguste Magnin (Geneva, 2013) is the project of reconstruction of the Geneva Plan-Relief. It has a high fidelity to the original, using a 3D scan, but has mainly been made manually. Rome Reborn (Dylla et al., 2010) is an interesting project of restitution of the Plastico Di Roma Antica that uses procedural modelling on the basis of scan data on certain areas: a program recreates plausible models, but not exact ones, in order to have the historic atmosphere of the city. It uses architectural rules from treatises translated to modelling language. Going further, a study on the automated generation of a Historical city Model of Hamburg has been done, including five different epochs (Kersten et al., 2012). It has been conceived by scanning acquisition on a scale model thanks to a robotic arm, and automation of reconstruction from maps. Finally, for the Plans-Reliefs exhibition in Paris in 2012, Google digitized many scale models in a short time, using pictures and laser scanning. Unfortunately, the laser scan data has only been used to verify buildings height, and pictures to create textures. Many graphic designers have modelled the Plans-Reliefs on SketchUp manually, with a loss of accuracy. Lastly, a PhD (Jacquot et al., 2013a) from our laboratory is being made on the plan-relief fortifications. The goal is to automate the adjustments of a parametric library of fortifications works thanks to reverse engineering process. In a previous paper (Chevrier et al., 2010), we explained how we managed to automate the modelling of the buildings from roof segments. However, these roof segments had been manually positioned with PhotoModeler, task that took a lot of time.

\subsection{Comparison between photogrammetry and 3D laser scanning}

As this paper is about 3D mesh acquisition from real models of cultural heritage, we had to care about the different technics of acquisition. Most of found studies are on real size objects. According to P. Grussenmeyer (Grussenmeyer et al., 2010) there are three methods: Laser Scanning, Photogrammetry, and Tacheometry. These methods have been tested on a full scale model: a castle. We can exclude tacheometry because of the small scale we are working on. Each of these has advantages and disadvantages: both capture heavy data with high precision; photogrammetry is easily textured and better detects edges. In the end, they seem complementary. Some researches insist on the fact that there is no method to be applied in every situation. They made some tests to select the most appropriate method and sometimes to combine them (Kadobayashi et al., 2004; Boehler et al., 2004; Skarlatos et al., 2012).

\section{3D ACQUISITION VIA PHOTOGRAMMETRY}

In this part, we present experiments that have been made on a 3D printed replica of a city block of the Toul plan-relief. In section 4.1., we compare several photogrammetrical software. Then, in section 4.2, we defined a protocol to take pictures according to the recommended advices of the user manual software. Finally, in section 4.3, we define a protocol for the 3D mesh creation in order to obtain a high quality mesh.

\subsection{Comparison of software}

We have been looking for a tool that would automate to the maximum the meshing process. Previously, we used Photomodeler Scanner, software that used photogrammetric methods in manual ways. Now that the technologies have evolved in this field, many software programs exist. We have tested several photogrammetrical software that use different algorithms and different protocols for taking pictures, to select the most appropriate to our need. To do it wisely, we followed the main instructions for each software program to have a proper set of pictures, rather than providing an identical set for all software. Elements of comparison have already been made, on real buildings (Grussenmeyer et al., 2008). We considered these researches to make our experiments, but the tested software often ask for manual intervention. Also, others photogrammetric programs appeared recently.

Tested softwares are: Autodesk 123DCatch, Agisoft Photoscan, EOS System Photomodeler. Other softwares, as Pistou (a research program in MAP-Gamsau, or Topcon's Imagemaster Pro have been considered, but we excluded them for their apparent complexity. Here are the results of our comparison; each one tested on the 3D printed scale model of Toul:

\begin{tabular}{|cccc|}
\hline Nb. Pict. & 123DCatch & Photoscan & Photomodeler \\
\hline Calc. time & $\approx 30 \mathrm{~min}$ & $\approx 30 \mathrm{hrs}$ & Manual \\
\hline $\begin{array}{c}\text { Quality of } \\
\text { the results }\end{array}$ & Good & Medium & Good \\
\hline Price & Free & $3500 €$ & $2500 €$ \\
\hline & & & \\
& & & \\
\hline
\end{tabular}

Table 1: Comparison of the different software.

As seen on table 1, 123DCatch asks for about 60 pictures, with a maximum of 80 . Photoscan needs pictures as much as possible, the user manual mentioning 100 to 500. Photomodeler is mainly usable manually, but an automation exists, which demands for couples of pictures, to work with stereogrammetry. After experiments, the most appropriate software to acquire high quality 3D meshes from scale models using photogrammetry appeared to be Autodesk 123DCatch. It has the quality of being free, fast, and offers easily exploitable results. On the other side, it leaves a very limited control to the user for its parameterization, and does not display any preview before it is completely calculated. So you have to wait for the results before knowing if the model is usable or not.

\subsection{Protocol for taking pictures}

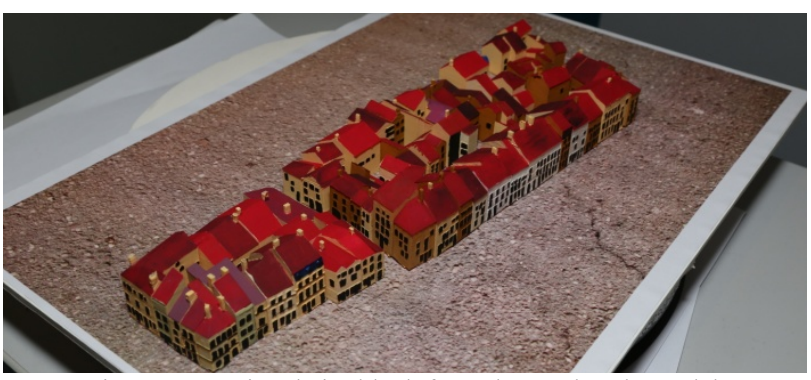

Fig. 1. Two printed city block from the Toul scale model.

To define the following protocol, we have tested different configurations in our laboratory. These tests were made on 3D printed city blocks of the Toul scale model, painted by hand and relying on a textured background (Fig. 1). The optimal configuration we found for taking pictures that are enough detailed and the most exploitable is a good single-lens reflex camera, equipped with a powerful flash device and its reflector. In the case of our study, we used a Canon EOS 6D, offering high quality pictures of 16 megapixels, equipped with a 24-70 lens. We had a laptop on the shooting place, to download and verify pictures. Also, we had the possibility to start a test with 123DCatch granting us a preview for exploitable results or not. 
Have been tested aperture, sensibility, shutter speed settings and different lightning conditions, with some help from a professional photographer. This protocol was only applied and verified on the Autodesk 123DCatch software. It has been provided by our own tests, but also by following the user manual, the user experiences and previous related works on photogrammetry in the Digital Heritage domain.

The Single-Lens Reflex is set on manual mode, assembled with the flash device and its reflector. The flash device allows us to take pictures even in the low luminous condition on which the scale models are often exposed. Its shutter speed is set to 200 milliseconds. It is a very short shutter speed, which makes the ambient light shadows disappear when synchronized with the flash device. The aperture is set up to 11 to have a good depth of field. Its ISO Sensibility is set on 800 , allowing us to take quite a lot of pictures without consuming too fastly the flash device battery (with a low quality loss compared to an ISO 100 setting). The Auto-focus is on, and the focal length is optimized according to the distance from the subject. The focal length has to remain the same for every picture on one unique subject.

The shooting conditions in which we work are not always great. Also, we tried to take pictures after verifying our own stability, and then to verify each picture. We have to choose parts of the subject of a reasonable size. The referent here is a city block and its near environment, as it can have in itself a real complexity. This represents an average surface of three square decimetres. Also, the distance to the subject has to be the shortest. In the first shoots, the subject must be entirely seen in every picture, and take the most of the area. Then, we can shoot scale model details. The nearest distance $(30 \mathrm{~cm})$ the littlest focal length (24), and the furthest distance $(1 \mathrm{~m})$ the largest focal length (70). We take two orbits of approximately 20 photos around the object. The first orbit at $30^{\circ}$ from the ground, the second at $60^{\circ}$ (Fig. 2). To complete this collection, we can add some photos orbiting around hidden areas. It will work better if there is a slight presence of volumes or a textured background all around the object instead of a solid colored one. We often arrive to a total of sixty pictures, which is generally enough to work with.

Furthermore, in order to have a spatial reference and scale for a future 3D mesh acquisition, it is advisable to place a small rule near the model. This will define a scale for it and make easier the following phases. After photographing the model following our protocol, we pass to the 3D acquisition phase with 123DCatch

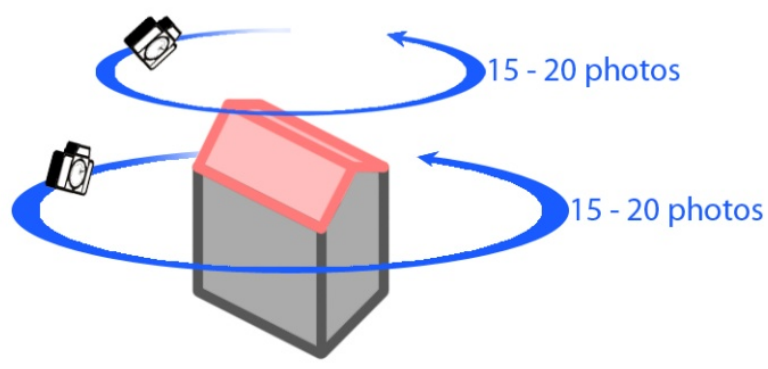

Fig. 2. Simple scheme for taking photos

\subsection{Protocol for the 3D acquisition with 123DCatch}

123DCatch is very simple to use. At the opening of the software, you only have to select the pictures you want to send. To simplify the computation, it can be useful to reorder the pictures by orbits. 123DCatch has a limit of upload of 80 pictures at a maximum resolution of 6 Megapixels. So we may have to resize the pictures before sending them. Then, we can send these pictures to the Autodesk cloud for calculation.
During this phase, no user manipulation is allowed. 123DCatch keeps running in the background, you can also close the program, asking Autodesk to send an e-mail when the mesh has finished calculation. Whatever the size of the photographed area, the number of polygons in the mesh will remain the same. The more you photograph closely, the more the mesh is accurate. Once the pictures are sent, the mesh is computed and downloaded. This step can take several minutes (between 10 to 30 minutes). We have for the first time a good preview of the calculated object. If the result is satisfying, you can continue working on it, otherwise you have to restart with others settings. At this stage, the program allows four different interventions: -Define common points between unused pictures and the used ones to add them correctly to the calculation.

-Add new pictures to clarify complex areas, making possible round-trips between photo shoots and 123DCatch.

-Give a precise scale to the model for a future exportation, hence the importance of taking pictures with a ruler.

-And above all, recalculate a more precise model on a selected area of the mesh. This is highly recommended, because the first computing is generally inaccurate.

After these steps, the model is ready to be exported. 123Dcatch has the advantage of offering many export file formats: *.obj compatible with many programs (will be used to export to Geomagic), *.fbx compatible with Autodesk Maya to eventually modify the $3 \mathrm{D}, *$.las which is a highly used point cloud file, and *.dwg compatible with AutoCAD.

Following this protocol, the acquired mesh quality is high and exploitable for next steps of treatment. Here are 123DCatch screenshots of these results on the Toul printed replica scale model (Fig. 3), and the real Epinal scale model (Fig. 4). As presented, meshes are well textured, and are accurate enough even in the centre courtyards. We always have small not captured details as they could not be seen from any point of view, as areas under the roof overhangs, small alleys $(<2 \mathrm{~mm})$, and the smaller and deeper courtyards $\left(1 \mathrm{~cm}^{2}\right.$ in $2 \mathrm{~cm}$ high courtyards).

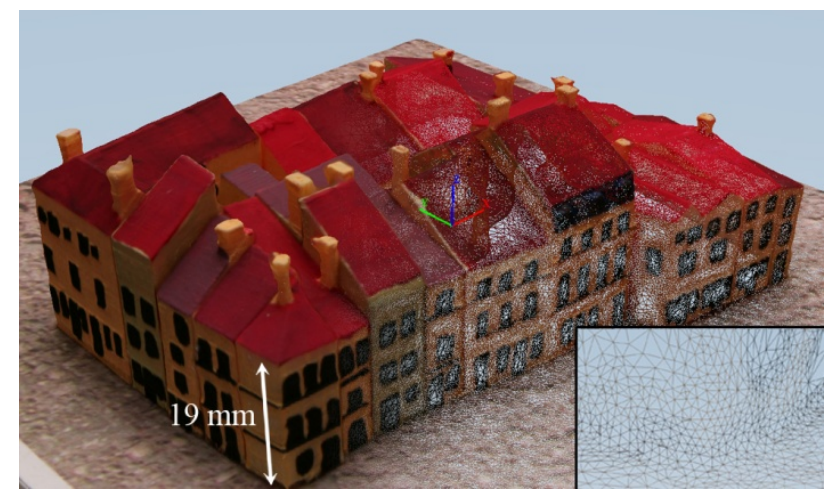

Fig. 3. 123DCatch results for Toul's replica scale model

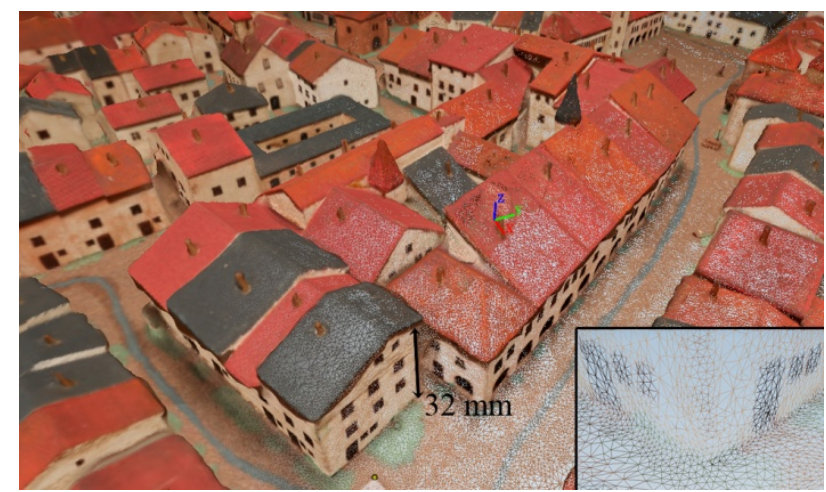

Fig. 4. 123DCatch results for the Epinal scale model 


\section{3D SCANNING ACQUISITION}

\subsection{Technical constraints}

Technical constraints due to the scale models and plans-reliefs are almost the same for scanning and photo shooting. Then, the access to the scale model, often difficult, is even more important for the 3D scan because of the necessity to sweep the model from many directions. Moreover, the 3D scan needs to be wired to electric power, and to a laptop. It becomes much more difficult to use than the Single-Lens Reflex in almost every case. The use of our handyscan (Creaform EXAscan) is easier on little scale models. Also, the handyscan needs special reference points, which consists on a grid marked with many points, every 5 centimetres.

\subsection{Tests}

To define the following protocol, we made many experiments in laboratory, always on the same 3D printed scale model of Toul, and also in real conditions, on the Epinal scale model. Experiments on the printed Toul scale model have been useful to define every settings of the scan (luminosity, sensibility, precision), and the scanning time. These settings are related to each other: we had to make a compromise between precision, scan time, and size of the bounding box. These settings have been used on another experiment, on the real Epinal scale model, where we tried to define how to scan in real conditions of work..

All these tests have been influenced by previous experiments from our laboratory. In the first experiments (Chevrier et al., 2010), previous settings have been already defined and tested in real conditions. These experiments conducted on Toul planrelief, have highlighted the difficulties to scan thin or damaged elements.

As special reference points, we suspended a wired doted grid over the scale model thanks to a portable scaffolding. This grid remains hardly installable over a massive scale model. We are not authorized to place pastilles on the model because it is forbidden by Cultural Heritage.

\subsection{Protocol for the 3D scan acquisition}

As the photogrammetry, 3D scanning needs some preparation. The device only scans what is in a radius of $30-45 \mathrm{~cm}$ from his sensors. Therefore, without an expensive robot arm to do this, the operator can only use his own arms, and count on the access he has to the scale model. As much as possible, it is recommended to study every access possibilities to the model, as if it is extremely rare to have a free access everywhere. The treated area by the scan is defined by its own acquisition quality settings. In the case of thin detailed scale models, the precision has to be high: one point each semi-millimetre. This setting limits the acquisition volume to a cube of $15 \mathrm{~cm}$ side. The full acquisition time for this volume with an accurate precision is about one hour. To scan the full model, you have to sweep over the model from many different points of view. The software displays in real time every acquired point, which allows us to control the missing areas. If there are areas that are inaccessible to the scanner sensors, it will leave a hole in the final mesh, which can be difficult to fill. In the case of very dark painted surfaces, it is possible to change the light sensibility setting of the scanner to avoid other holes. The scanner cannot acquire areas painted that are near to the absolute black (in our case the openings of the buildings), which absorbs every light.

With laser scanning, we have been able to acquire high quality $(0,2 \mathrm{~mm}$ in our case) non-textured meshes, similar in quality to the photogrammetry. Each different acquisition is geo- referenced due to the dot grid, making easier the assemblage between them. Also, captured models are already at scale, which is a benefit for further operations.

We present the results of each scan on the Toul printed replica scale model (Fig. 5), and then on the real Epinal scale model (Fig. 6). The meshes are of good quality, but there are always some holes corresponding to parts that the scanner could not capture. But no textures are available. Earlier tests have been made with a textured scan, but results were not convincing. Textures are very rough, and they are not captured on the whole scale model, so they are not exploitable. For the Epinal acquisition, we focused on a part of the model that was easily accessible on the border.

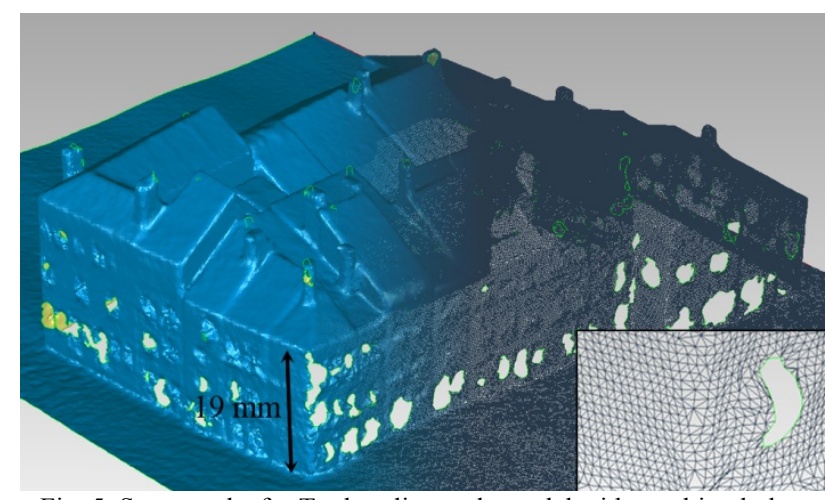

Fig. 5. Scan results for Toul replica scale model with resulting holes from non-captured black surfaces.

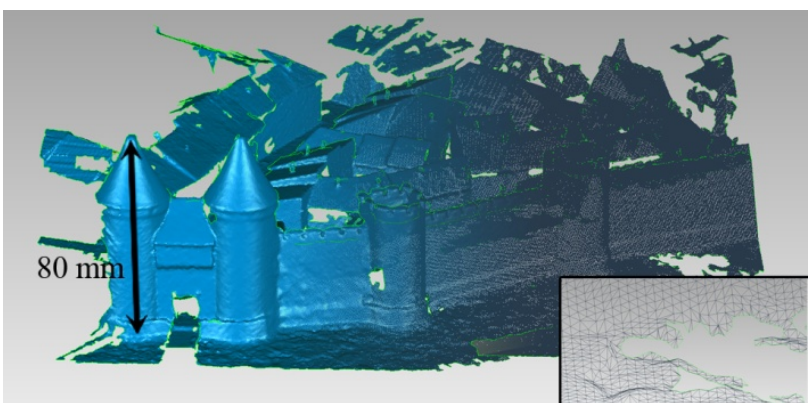

Fig. 6. Scan results for Epinal scale model: the back sides are missing because it is not accessible by the hand.

\section{COMPARISON BETWEEN PHOTOGRAMMETRY AND 3D SCAN}

In order to know which method is the most accurate in the case of city scale model, we compared each method results. We compared the mesh quality (section 6.1), the acquisition difficulty (section 6.2), the exploitability and the eventual extras that they offer (section 6.3).

\subsection{Mesh quality}

The mesh quality is important for us, as we need a high quality to exploit the model in order to segment it. Considering the amount of polygons as a part of a mesh quality, the results between photogrammetry and laser scan are similar (Fig. $3,4,5,6)$. In comparison, each scanned cubic areas $(15 \times 15 \mathrm{~cm})$ has 1.4 million polygons, whereas every 123DCatch mesh of the same size has up to 1.6 million polygons (depending on the number of pictures and their resolution). The mesh quality of the photogrammetry depends on the area we want to acquire and on the resolution of taken pictures, whereas the quality of the scan depends on the amount of defined sized cubic areas.

The mesh quality is not just about the polygons number. Others characteristics come into play: the accuracy of the acquired 
geometry, and the precision on details. On these criterions, laser scan has less noise on solid coloured parts, but photogrammetry is still enough for segmentation, and both cannot access in thin holes. Taking detailed pictures for the photogrammetry, we can improve geometry.

The available results in the fortified areas of the scale models are along the same line. Even if the photogrammetric mesh is more irregular than the laser results, the impact on the segmentation process remains invisible.

\subsection{Difficulties of acquisition}

Because of the size of scale models, we had to find the most convenient method to acquire its geometry. As a reminder, Epinal scale model, one of the smallest, is $3.3 \times 1.8 \mathrm{~m}$. The only city table of Toul is already $2.3 \times 2.13 \mathrm{~m}$. So, some areas can be difficult to access (Fig. 7). Moreover, given the little control over the parameters of 123Dcatch, it is impossible to forbid its algorithms to create awkward geometries instead of trees and areas it could not capture properly. Therefore, photogrammetry is easier, thanks to the zoom and focal we can modify according to the distance we have to the subject. The Single Lens Reflex is also a lighter device than the Laser Scan. The Laser Scan has the disadvantage of needing a specific distance between it and the subject, which can often be difficult to access. This is resulting by holes and missing parts on the mesh (Fig. 6). This disadvantage could be bypassed by the use of a robotic arm or a bridge over the model, of a consequent price. The additional cost of these operations includes a truck, the equipment (the LaserScan we used costs about $55000 €$, and the Single-Lens Reflex $3000 €$, which is much cheaper), and the loss of time from moving and installation.

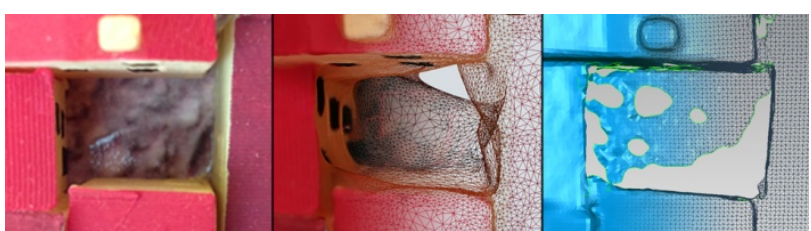

Fig. 7. Detail of a small and deep courtyard: on the left, the real scale model; in the middle, 123DCatch finds an average round geometry; on the right, the scan leaves holes instead of proposing a fake geometry.

\subsection{Exploitation possibilities and extras}

The exploitation possibilities offered by each method are similar. Thanks to the accuracy of the laser scan, segmentation part can be a bit easier than the photogrammetry, because of the slight noise on 123DCatch results. Scan reorganization is also easier thanks to the geo-referencing of each captured mesh, but it is also possible with photogrammetry in Geomagic Studio 12. In contrast, photogrammetry offers well textured meshes exploitable for the next steps.

\subsection{Chosen method}

After this comparison, we chose to give priority to the photogrammetry, because of its ease of use, its rapidity in situ, its price, and the needed capacity of texturing the models.

\section{SEGMENTATION AND PLANES RECOGNITION IN THE 3D MESHES}

After acquiring meshes from the whole scale model, we have many separated point clouds. These point clouds are heavy (about 1 million points per city block) and represents a very complex geometry composed of many polygons which are too heavy to be viewable online. So we have to treat this point cloud with respect to building shapes and roofs, in order to have a lighter model. The aim of plane recognition is to facilitate an automatic parametric modelling (that will be explained in a another paper). The texturing of the parametric model is automatically computed from the textured mesh.

In section 7.1, we compare software applications for segmentation. Then, in section 7.2, we define a protocol for this step. And finally, in section 7.3, we will see the final results of segmentation.

\subsection{Comparison of software}

In the domain of segmentation of 3D meshes, many software applications exists. We decided to test three software programs to evaluate which one is the most adapted to our needs. Many of these are commercial and expensive, because of their rarity and complexity. Tested software includes Geomagic Studio 12, Rapidform XOR3, EDF CloudCompare and Mesh2Surface 4 Rhino plug-in. EDF CloudCompare is free, it applies RANSAC algorithms with medium quality results, but it offers very low export possibilities. Mesh2Surface 4 Rhino is a Rhino plug-in that costs around $600 €$. It has been tested on its demo version, and did not offer exploitable results. Rapidform and Geomagic are recognized among the best point-cloud processing software. Their price is high $(15000 €)$ and their functionalities are very close to each other. However, Geomagic Studio 12 is an easyto-use software that offers great exploitable results. It has many parameters that allow the refinement and the enhancement of the meshes, to prepare them to the phase of segmentation. Also, many of the operations are automated, and never ask to interfere directly on the mesh. That's why we decided to use Geomagic Studio 12 .

\subsection{Protocol for the segmentation}

In order to segment the meshes, we will describe the use of Geomagic Studio 12, step by step. The general way to operate the mesh is: first, correction of the mesh, in order to avoid any calculation error, then optimization of the mesh, in order to simplify the computing in the following steps, and finally mesh segmentation. Each of these steps asks for many parameters, that we have to define. We have to find the optimal default values to have the best conditions to automate the process. Let us detail the three steps:

- Many functions help to correct the mesh. The first one is automatically proposed by the program, called the "Mesh Doctor". This operation reduces little spikes, holes, replaces the normal of false oriented triangles, and also improves the mesh quality in order to avoid any error on next steps. Others manual functions let the user complete the correction step, as hole filling, mesh trimming, etc. In the fortified areas, this process is a tedious task because of the vegetation cover which has to be removed. This operation can only be done manually given the proximity of the vegetation with the smallest details of the fortification works. Even with these precautions, the loss of information is inevitable and prejudicial for the segmentation step. Thus, it results in many program crashes delaying the segmentation process.

- Then, we prepare the mesh for the segmentation, by cleaning low curvature areas, reducing noise, and sharpening. These options are facultative, but they help to the segmentation, especially on imprecise meshes.

- Finally, the parametric surfacing / segmentation phase, finishes our process. This step passes by many actions. First, the region detection asks the user to enter sensibility parameters. Different parameters are asked at this time, as the separator and curvatory sensibility, and the minimum area to be computed. 
Obviously, it often depends of the mesh quality, and the nature of the model. In the case of city blocks: sensibility must be high, and the minimum area is about a half square centimetre. Next, we define the type of geometrical shape we want to recognize (Planes for building reconstruction, Sphere for spherical trees, Freeform for ground). These regions are separated by their contours, which the user is able to modify and correct. In the fortified areas, manual interventions are always necessary as the fortified entities vary greatly in terms of size. The walls are usually correctly segmented depending of the amount of residual noise but smallest parts of fortification are nearly out of range even with a high sensibility and a minimum area about of a tenth of a millimetre.

Almost every face connection on scale models are edges, so we set the connexion type on "sharp". Then comes the phase of surfaces and connections fitting. And finally the result computing, that offers a real segmentation, separated in trimmed primary surfaces. By following these steps and settings, we have a segmented model, approaching the real one, and allowing us further automatic treatments for the creation of the parametric models of the buildings (Fig. 8).

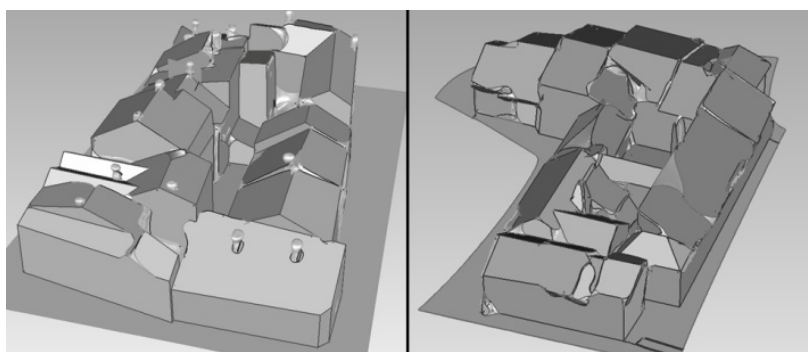

Fig. 8. Results of segmentations from Geomagic for Toul replica scale model, and for the real Epinal scale model

\subsection{Results}

Thanks to the plane recognition, the model becomes simpler, only composed of real plane polygons following their real edges. There are still some improvements to do, such as missing corners that needs even more precision, and the presence of chimneys that can be easily found (they are formed by spikes). In the fortified part of scale models, the level of detail is higher than in the city area as the plans-reliefs were used above all as a military tool. Geomagic treatments and especially segmentation step are time consuming processes whose outcomes are far from being satisfactory. An alternative method is being tested right now. It uses Grasshopper plug-in for geometric and semantic segmentations and the early results are encouraging (Jacquot et al., 2013b).

\section{CONCLUSION}

This study about the use of photogrammetry and laser scanning on historical city scale model has revealed some important knowledge about the protocols for acquisition, and segmentation. From the different sizes of the scale models, compositions, and epochs, we could define a unique protocol to have the same quality of results. Tests have been focused on the city and fortifications, but they have to be extended to towers, vegetation, historical monuments, etc.

The next step of our researches is in progress: the automation of the creation of the building parametric models from the $3 \mathrm{D}$ segmented meshes. The texture of each building will be computed from the 3D textured mesh. The benefit of that kind of semantic and digitalized city model would be great: it will be accessible to the public; it will represent an information source useful for historians, patrimonial architects and town planners; it will promote the scale model collections to everyone.

\section{ACKNOWLEDGEMENTS}

Thanks are due to the photographer Gilles André of the SRI (Service Regional de l'Inventaire de Lorraine) for his advices.

\section{REFERENCES}

Boehler, W. and Marbs, A., 2004. 3D Scanning and photogrammetry for heritage recording: a comparison. Geoinformatics 2004, University of Gävle, Sweden, 7-9 June 2004

Chevrier, C., Jacquot, K. and Perrin, J., 2010. 3D Modeling of a town scale model. Limassol, Cyprus, pp. 99-107. LNCS 6436.

Dylla, K., Mueller, P., Ulmer, A., Haegler, S. and Frischer, B., 2009. Rome Reborn 2.0: A framework for virtual city reconstruction using procedural modeling techniques.

Geneva, 2013. Scale model of geneva, project pram. http://youtu.be/MwLDoZnv7oA (accessed june 2013).

Grussenmeyer, P., and Al Khalil, O. 2000. A comparison of photogrammetry software packages for the documentation of buildings. The Mediterranean Surveyor in the New Millennium Seminar, 18-21 September 2000, Malta.

Grussenmeyer, P., Landes, T., Voegtle, T. and Ringle K. 2008. Comparison methods of terrestrial laser scanning, photogrammetry and tacheometry data for recording of cultural heritage buildings. The International Archives of the Photogrammetry, Remote Sensing and Spatial Information Sciences. Vol. XXXVII. Part B5. Beijing, China.

Jacquot, K., Chevrier, C. and Halin, G. 2013. Validation of a parametric approach for 3D fortification modeling: Application to scale models. 5th International Workshop 3D-ARCH'2013: $3 D$ Virtual Reconstruction and Visualization of Complex Architectures. 25-26 feb. 2013. Trento. Italia.

Jacquot, K., Chevrier, C. and Halin, G. 2013. Reverse Engineering of Scale Models Using Dataflow Programming:: Application to the fortification of plans-reliefs. Digital Heritage. 28 oct-01 nov. 2013. Marseille, France. (submitted)

Kadobayashi, R., Kochi, N., Otani, H. and Furukawa, R. 2004. Comparison and evaluation of laser scanning and photogrammetry and their combined use for digital recording of cultural heritage.

Kersten, T.P., Keller, F., Saenger, J. and Schiewe, J. 2012. Automated generation of an historic 4D city model of Hamburg and its visualization with the GE Engine.

Skarlatos, D. and Kiparissi, S. 2012. Comparison of laser scanning, photogrammetry and SFM-MVS pipeline applied in structures and artificial surfaces. ISPRS Annals of the Photogrammetry, Remote Sensing and Spatial Information Sciences, Volume I-3, 2012 XXII ISPRS Congress, 25 August01 September 2012, Melbourne, Australia 